\title{
Electron density, and its interplay with the energy and properties of molecules and solids
}

\section{J. Contreras-García}

\author{
CNRS-Sorbonne Universités, Laboratoire de Chimie Théorique, Paris, France \\ julia.contreras.garcia@gmail.com
}

Electron density has been a great source of insight in the understanding of bonding and structure. Nonetheless, it lacks a fundamental characteristic: its connection to molecular and solid properties is barely predictive. This is so due to the lack of a direct (known) link between electron density and energetics.

Along this contribution we will try to fill this gap for several relevant cases in crystallography.

One way to approach this gap is to build energy models relying on topology. We have explored using a potential energy surface that includes chemical quantities explicitly, so that properties provided are directly related to the inherent organization of electrons within the regions provided by topological analysis [1]. Coupling this to conceptual DFT, the band gap of solids can be univocally defined [2]. Applied to zinc-blende solids as a model case, trends in band gap can be predicted in terms of bond properties (length, charge, crystalline structure- Figure 1).

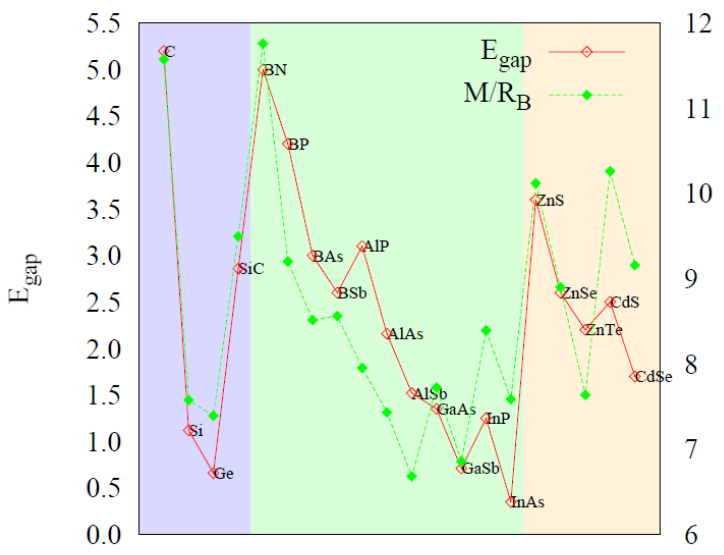

Figure 1. Band gap (in eV) for IV (purple), III-V (green) and I-VI (light orange) compounds from experimental data and from our model.

Another field where energetic estimates from the electron density are missing is molecular crystals. This kind of approach would reveal extremely useful to predict the stability of different molecular crystal polymorphs or even cocrystals. In order to build this knowledge, we rely on the Non-Covalent Interactions (NCI) index, which is able to identify the regions relevant to weak interactions from the electron density alone [3]. Simple approaches for well-known intermolecular energies datasets have allowed us to show that the energy can be predicted from these electron density regions using machine learning approaches in a fast and accurate manner [4]...next step is crystals!

[1] R. F. Borkman, R. G. Parr (1968) J. Chem. Phys. 48, 1116.

[2] J. Contreras-García, C. Cárdenas (2017) J Mol Mod 23, 271.

[3] E. R. Johnson, S. Keinan, P. Mori-Sanchez, J. Contreras-García, A. J. Cohen, and W. Yang, (2010) J. Am. Chem. Soc. 132 , 6498.

[4] F. Peccati, E. Desmedt, J. Contreras-García (2019) Comp. Theo. Chem., 1159, 23.

Keywords: topology; ELF, NCl; electron density-properties relationships; band-gap; stability 